S

\title{
Recherche en stratégie : un problème de granularité
}

La complexité des situations stratégiques est reconnue par tous les décideurs. Elle appelle donc logiquement une pensée, des concepts et des outils aptes à embrasser cette complexité, dotés d'une granularité adéquate. Mais le courant dominant préfère continuer à établir des micro-relations de causalité simples - si $A$ alors $B$-, souvent pointillistes et extraites des contextes. Ce faisant le corpus se détourne de sa raison d'être : produire des savoirs d'action, des heuristiques dotées d'une granularité adéquate, à même d'aider les décideurs à rendre intelligibles leurs situations stratégiques, au profit d'un scientisme de plus en plus éloigné des questions du moment. Pourtant la longue et robuste tradition de l'épistémologie pragmatiste, en Occident comme en Orient, offre un paradigme alternatif beaucoup plus en accord avec la raison d'être et les spécificités de la stratégie. 


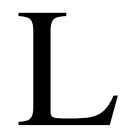
a stratégie se pense et se met en acte parce que des situations font problème. Situations évolutives, où l'abondance et la parcellisation des données et des symptômes côtoient souvent un déficit de repères et de sens, des préférences de décideurs ambiguës et diverses, où les buts et les moyens s'enchevêtrent et se produisent réciproquement...bref, des situations mal structurées (Simon), magmatiques (Castoriadis), complexes (Morin).

Pour le stratège en acte, la complexité de la stratégie et des situations qui l'appellent ne fait aucun doute. Et c'est bien cette complexité qui a suscité le remarquable effort de recherche des années 1960, véritable décennie de la stratégie, avec les textes fondateurs d'Ansoff, Andrews, Chandler. Fondateurs mais oubliés des chercheurs malheureusement, alors qu'ils se coulaient dans une épistémologie appropriée à l'objet et au projet de connaissance, très spécifiques, de la stratégie. Il s'agit en effet de produire des savoirs d'action, rigoureux certes, mais d'abord pertinents pour des décideurs, en leur proposant des cadres conceptuels, des schémas directeurs, des heuristiques, à même de guider leur réflexion tout en les incitant à " remplir les blancs », à introduire la singularité inexorable de leur situation.

Poursuivie par les auteurs majeurs du champ - Mintzberg, Porter, Prahalad, Ghoshal, Quinn, Van de Ven, Miller... -, cette production de connaissances génériques, aptes à favoriser l'engendrement du nouveau a, en conséquence des injonctions du colloque de Pittsburgh en 1978, été délaissée et bientôt dénigrée par le flot de publications dominant, où la quête de reconnaissance académique et le mimétisme méthodologique consacrent le souci de
« faire science à propos de la stratégie » plutôt que d'amplifier le répertoire des savoirs mobilisables dans et pour concevoir l'action stratégique (Martinet, 2007).

\section{I - « LA COMPLEXITÉ APPELLE LA STRATÉGIE »}

Cette désormais célèbre assertion d'Edgar Morin, élaborée tout au long de son œuvre monumentale, La Méthode, s'adresse d'abord à l'acteur: «Il n'y a que la stratégie pour s'avancer dans l'incertain et l'aléatoire (...), elle est l'art d'utiliser les informations qui surviennent dans l'action, de les intégrer, de formuler soudain des schémas d'action et d'être apte à rassembler le maximum de certitudes pour affronter l'incertain » (Morin, 1990, p. 178).

Quel que soit son statut, sa taille ou son activité, une organisation est toujours unitas multiplex, inexorablement plurielle et composite, que la stratégie s'efforce de constituer en unité, sans jamais y parvenir complètement...sauf à la faire devenir totalitaire. L'acteur stratégique vise à imposer des règles du jeu et des normes qui lui paraissent favorables au devenir de l'organisation, mais doit composer sans cesse avec les réactions et les projets des autres, de tous ceux qui cherchent à opérer dans le même espace ou des espaces intersectés. La tension dynamique, le plus souvent loin de l'équilibre, entre autonomie et hétéronomie constitue le principe premier de la stratégie, toujours produite en contexte, flux d'actions jamais indépendantes de leur écologie, sauf à franchir des seuils critiques et pathologiques, comme le montrent à l'envi toutes les tentations et mauvaises pratiques (Denis et Martinet, 2012) de management « hors-sol ». 


\section{II - « LA STRATÉGIE APPELLE DONC UNE PENSÉE COMPLEXE »}

Complexité informationnelle, computationnelle, de sens, nécessitant une rationalité procédurale interactive, une raison dialoguée irréductible à une rationalité $a$ priori et unique, qu'elle soit financière, technologique ou autre. Et donc complexité cognitive, communicationnelle et politique, appelant une pensée-méthode suffisamment complexe et souple à son tour, largement conceptuelle et qualitative, favorisant les itérations entre le local et le global, les données et les interprétations, les symptômes et les diagnostics, l'articulation des différentes dimensions. Une pensée de l'intégration donc (Volberda, 2004), tendue vers le «comprendre suffisamment pour concevoir ce qui n'existe pas encore", connaissance fort éloignée de ces lois élémentaires censées expliquer ce qui est déjà là, et auxquelles il suffirait de se conformer pour réussir.

Et pourtant le courant dominant privilégie la publication de ces « micro-lois », complaisamment autoproclamées «contributions théoriques » qui, comme le déploraient il y a quinze ans déjà, deux chercheurs majeurs du champ, Tsoukas et Knudsen, n'analysent qu'un monde passé, déjà là, posé comme fini, immuable et pré-ordonné, dont l'explication vaudrait prédiction, à l'instar de la mécanique du $19^{\mathrm{e}}$ siècle, mais dispensant ici le chercheur de tout prolongement technique ou praxéologique, les "implications managériales » par lesquelles il ne manque pas de conclure ses articles, n'étant le plus souvent qu'une redite tautologique et triviale, souvent simpliste, de ses «résultats ». Malheureusement, en ce domaine, les explications locales et statiques ne valent pas prédiction, ni même prévision, encore moins conception, et s'avèrent d'un piètre secours pour les praticiens...qui ignorent même l'existence de la plupart des revues académiques... et ne s'en portent peut-être pas plus mal. À cet égard la Revue française de gestion, avec son remarquable impact tel que mesuré par Google, fait partie des exceptions. Et il est significatif que ses articles les plus lus ou cités sur ses 40 ans d'existence ne relèvent pas de cette catégorie et présentent notamment une granularité plus large, ce qui en soi constitue un critère pour apprécier la pertinence d'un travail de recherche en stratégie.

Pertinence dont se désintéresse le courant dominant, comme le confirmaient de façon judicieuse mais déprimante, deux autres auteurs majeurs du champ, Bennis et O'Toole, dans une autre revue soucieuse de l'action, Harvard Business Review: « Nous avons accumulé une vaste décharge de publications 5 étoiles, en laissant à d'autres le soin des applications ».

\section{III - « E PUR SI MUOVE... »}

Sauf à renoncer à sa raison d'être, à se cramponner à une épistémologie incongrue, la recherche en stratégie ne peut durablement se fourvoyer à brosser $\mathrm{cm}^{2}$ par $\mathrm{cm}^{2}$ un monde passé, sédimenté, enterré, comme le ferait un archéologue, pour en expliquer des performances elles-mêmes dépassées, et ce d'autant moins qu'elle occulte délibérément l'historicité de son objet, tout obsédée qu'elle est de dégager des lois généralisables, extraites du temps et de l'espace, condition rhétorique certes nécessaire pour laisser croire à leur validité prédictive, quand bien même elles en sont le plus souvent dépourvues épistémologiquement. 
Alors même que le monde des affaires et les sociétés changent à grande vitesse et que la stratégie, comme «indiscipline » praxéologique, n'a d'intérêt que si elle aide à mieux concevoir d'autres voies et moyens, d'autres pratiques, efficientes et efficaces, mais tout autant responsables et soutenables pour le devenir des organisations (Tannery, 2014). Certes les modèles d'affaires disruptifs, la révolution numérique, mais aussi la corruption, les organisations maffieuses ou pirates, l'esclavagisme moderne, la domestication de l'homme ... commencent à intéresser quelques bons chercheurs du champ, mais ce dernier rechigne dans son courant dominant à s'emparer pleinement de ces nouvelles questions stratégiques. Les stratégistes militaires seraient pourtant condamnés au silence, s'ils n'ajoutaient pas aux guerres conventionnelles et à la dissuasion nucléaire, la guérilla, le terrorisme, les proto-États, les guerres climatiques...Faute d'un aggiornamento exigeant, trop de stratégistes d'entreprise se condamnent eux-mêmes à ne pas être audibles en se cramponnant à l'analyse fragmentée d'un monde passé.

De même façon, la recherche en stratégie doit en effet tirer les implications épistémologiques et méthodologiques de la multitude et de la fluidité, de l'accélération du temps des affaires humaines et des sociétés, de la destruction... pas toujours créatrice, de l'entrée dans l'anthropocène, des effets pervers de l'emballement technologique, des pratiques managériales produisant l'homme inutile ou dévasté...

Urgente invitation donc à ouvrir les objets et les projets de connaissance contre le consensus auto-satisfait et débilitant sur un objet et des méthodes embaumés (Nag et al., 2007). Urgence du pluralisme épistémologique et méthodologique nécessaire à la production de savoirs d'action et donc inscrits dans une philosophie pragmatiste, à une extension et à un renouvellement du domaine de la stratégie (Tannery et al., 2014). Bref à refaire de la stratégie pour les temps présents et à venir, comme nous y invitons avec quelques collègues depuis longtemps déjà (Desreumaux, 2008 ; Martinet, 2008, 2009 ; Hafsi et Martinet, 2007).

\section{IV - LA STRATÉGIE COMME ÉMERGENCE GUIDÉE}

Les pratiques actuelles des organisations innovantes, a fortiori celles dont la recherche en stratégie doit guider l'imagination et la conception, ne relèvent plus du paradigme de la mécanique classique et de l'épistémologie positiviste auxquelles se réfère, de façon plus ou moins explicite et consciente, la majorité des publications dites scientifiques. Ni des quatre principes du discours de la méthode de Descartes, qui conduisent lentement mais sûrement la recherche en stratégie dans l'impasse encombrée de l'insignifiance.

La stratégie doit certes continuer à élaborer aussi rigoureusement que possible des repères, des critères d'action, mais ne peut plus se réfugier dans le seul déroulement d'un plan et d'un programme, pas plus qu'elle ne peut s'appuyer sur un corpus de recherche qui ne serait que la juxtaposition de micro-lois, en espérant que celle dont on a besoin a bien été établie avant qu'un jour - le $7^{\mathrm{e}}$ bien sûr ! - tout le monde puisse se reposer et contempler la reconstitution $\mathrm{du}$ grand puzzle ou du «plan de câblage » (Le Moigne) que le Grand Créateur du Monde Organisationnel aurait malicieusement enfoui sous les pavés ou la plage et 
auquel chacun aura de façon ultra-parcellaire, contribué, permettant... enfin... le pilotage automatique par ce grand et définitif logiciel.

La stratégie relève plus que jamais du déséquilibre dynamique, de l'assimilationaccommodation (Piaget), de la composition permanente, des luttes-coopérations entre intentions délibérées et émergences imprévisibles, du téléologique et de l'écologique - respectivement pli grec et propension chinoise si l'on se réfère à François Jullien -, des ilots de certitude dans l'océan expansif des incertitudes, des concepts et de la raison mais tout autant des affects et de l'émotion, et nécessite que les éléments de connaissance soient resitués dans des modélisations englobantes, des frameworks, fussent-ils condamnés à n'être que des esquisses de schémas directeurs en perpétuelle élaboration. Pour voir plus loin et mieux, conseillons au stratégiste comme au stratège de préférer, parmi les épaules de géants sur lesquelles se hisser, celles de Spinoza, Vico et Pascal plutôt que de Descartes ou Laplace. Et d'assumer, de façon fort stimulante d'ailleurs, la fulgurance définitive de l'auteur des Pensées: « Je tiens pour impossible de connaître le tout sans connaître les parties, pas plus que de connaître les parties sans connaître le tout »(Pensées, p. 31), pierre systémique jetée sans doute avec délectation par le grand Janséniste dans le jardin à la française de ce « Cher René » qu'il jugeait incertain et inutile.

Toute organisation que la stratégie prétend guider étant toujours cette « auto-éco-ré-organisation » conceptualisée par Edgar Morin, il est dès lors impossible, pour le chercheur désireux de proposer des savoirs pertinents pour la conception de pratiques innovantes, de ne pas cultiver le caractère irréductiblement dialogique de la stratégie. On ne trouvera de théorie générale de la stratégie ni chez Porter, ni dans la Resource Based View , ni dans quelque théorie de moyenne portée qu'il conviendrait de transformer en grande théorie - pour reprendre les catégories célèbres du sociologue des sciences Robert Merton -, n'en déplaise à Sanchez et Heene (2010) puisque le problème est ailleurs. Mais faute de cette introuvable théorie générale, d'un impossible et d'ailleurs inintéressant « $\mathrm{e}=\mathrm{mc}^{2} »$ propre à la stratégie, posons que la dialectique généralisée - ou une Dialogique incluant les trois principes fondamentaux de la pensée complexe de Morin : dialogique, récursivité, hologramme - fournit un paradigme, une matrice d'élaboration de connaissances rigoureuses et pertinentes, parfaitement congrus avec l'objet et le projet de la stratégie qui ne vaut que par les savoirs d'action qu'elle propose et non par les moyennes et les variances qu'elle calcule sur des échantillons soi-disant représentatifs, résultats sans grand intérêt quand il s'agit de stratégies d'entreprises à qui l'on prêche par ailleurs de cultiver la singularité ?

\section{V - LA STRATÉGIE COMME CONNAISSANCE ÉTHIQUE ET PRAGMATIQUE}

Délaissons l'agitation et les fausses oppositions, artificiellement entretenues par le réductionnisme cartésien et le respect intégriste du tiers exclu et de la logique aristotélicienne. Que d'articles laborieux pour essayer de trancher l'indissociable planning vs learning, analyse de l'industrie 
vs approche par les compétences, leadership $v s$ stratégie... - et réjouissons-nous de voir aujourd'hui un chercheur de tout premier plan comme Nonaka, appeler à un tournant pragmatiste de la stratégie (Nonaka et Zhu, 2012). Et rejoindre ainsi ce que nous avons modestement tenté dans un programme de recherche de plusieurs décennies (Denis et al., 2011), en érigeant aussi l'évolution guidée en principe premier de la stratégie, avec Lovas, Ghoshal, Hamel, Prahalad et quelques autres.

En posant la stratégie comme contingente, cheminante, continuée, co-créative, collective et courageuse - «Les 6C»-, ils proposent une modélisation heuristique et non ontologique de la stratégie en s'inscrivant dans une philosophie pragmatiste où dialoguent l'Occident et l'Orient, qui défend une connaissance scientifique active, instrumentale et transformatrice, en lieu et place de la connaissance passive, spectatrice et représentationnelle que revendique le positivisme et que continue à produire le courant dominant en stratégie.

Il s'agit moins d'expliquer des relations élémentaires, de les extraire de leur contexte pour les qualifier de lois à portée générale, que de fournir des opérateurs de sens, des instruments pour rendre intelligibles des situations mal structurées et indéterminées faisant problème pour la vie des hommes en société, avant de proposer des voies et moyens pour les transformer, non sans avoir clairement affiché les finalités et les valeurs poursuivies. Attitude scientifique qui rejoint celle d'Albert O. Hirschman, pratiquée tout au long de son œuvre immense, désireux qu'il était de " dégager des possibilités qui ne sont pas des nécessités » (Martinet, 2012).
Plutôt que de lois générales expliquant des causalités simples sur des données empiriques passées mais extraites de leur historicité, la stratégie a besoin de propositions génériques élaborées via une double enquête scientifique, empirique et conceptuelle, orientées par une éthique explicite, et de nature à faire advenir ce qui n'existe pas encore (Martinet et Payaud, 2010), condition d'une science sociale féconde écrivait d'ailleurs Hayek, grand critique de ce scientisme qui guette toujours les sciences de l'homme et de la société.

Se pose donc en sciences de gestion, et singulièrement en stratégie, un problème majeur : la granularité de la connaissance. «Ce qui est simple est faux, ce qui ne l'est pas est inutilisable » écrivait Paul Valéry chez qui on a moins vu l'épistémologue que le poète. Nous serions tenté de dire que la recherche en stratégie a été bien trop productrice d'énoncés « vrais » mais inutilisables parce que simples voire simplistes, de plus en plus pointillistes plutôt que pointus, alors qu'il lui faut produire des formes et des configurations complexes mais utilisables qui, tout en reposant sur des données empiriques riches mises en tension avec des concepts adéquats, cherchent moins à dire le «vrai », à établir l'explication ultime, qu'à offrir des appuis valides à la conception de stratégies nouvelles. Taxonomies, frameworks, systèmes propositionnels, grammaires... fournissent en effet pour les stratèges, les formes actuelles des «tableaux de pensée » que produisait un autre géant, Max Weber, et des heuristiques, beaucoup plus puissantes et pertinentes, fussent-elles qualitatives, que des micro-lois - Si A alors B - dont le grain est presque toujours trop fin pour éclairer une situation stratégique et dont la 
prétention universalisante est contreproductive tant le contexte est capital en stratégie. Il est d'ailleurs piquant et réconfortant de constater que le chercheur en stratégie le plus fécond, le plus cité et le plus récompensé de ces dernières décennies, Danny Miller, produise essentiellement des configurations, des formes et des typologies tout à la fois empiriquement fondées et éclairantes pour susciter et éclairer des décisions stratégiques à venir (Miller, 1996 ; Miller et Le Breton-Miller, 2010). Dommage qu'il ne soit pas davantage imité dans ses démarches alors même qu'il publie dans les revues les mieux classées ; cela suppose il est vrai des compétences distinctives.

À regarder de près le numéro anniversaire des 40 années de la Revue française de gestion et la judicieuse introduction de ses derniers rédacteurs en chef (Barthélémy et Denis, 2015), on relèvera, non sans quelque satisfaction, que les articles les plus lus, les plus cités, les plus appréciés sur cette longue période, présentent quasiment tous une certaine complexité, une qualité de mise en perspective, un effort synoptique sinon de synthèse, bref une granularité assez large, qui n'est sans doute pas étrangère à leur succès et à leur impact, y compris au-delà du monde académique. À l'inverse de ces articles qui, pour faire science, cherchent à dire tout sur presque rien, et dont on voit de moins en moins comment dans nos domaines, ils pourraient contribuer à ce que les hommes vivent un peu mieux, via des pratiques managériales plus pertinentes, responsables, soutenables, éthiques et davantage respectueuses des contextes de leur déploiement.

\section{BIBLIOGRAPHIE}

Barthélémy J. et Denis J.-P. (2015). « Les articles les plus influents de la Revue Française de Gestion », Revue française de gestion, vol. 41, $\mathrm{n}^{\circ}$ 253, spécial anniversaire « 40 ans d'influence », p. 7-20.

Denis J.-P., Martinet A.C., Payaud M.A. et Tannery F. (2011). Gouvernance et Stratégies des Groupes, Paris-London, Hermès-Lavoisier.

Denis J.-P. et Martinet A.C. (2012). « Le management, un carré plus que sémantique », Revue française de gestion, $\mathrm{n}^{\mathrm{o}}$ spécial 228-229, p. 13-38.

Desreumaux A. (2008). « Refaire de la stratégie », FCS, vol. 11, juin, hors-série, pp. 67-108.

Hafsi T. et Martinet A.C. (2007). «Stratégie et management stratégique : un regard historique et critique », Gestion, vol. 32, $\mathrm{n}^{\circ} 3$, p. 88-98.

Martinet A.C. (2007). «Savoir(s), connaître, agir en organisation : attracteurs épistémiques », La Construction de Savoirs pour l'Action, Avenier M.-J., Schmidt C., Paris, L'Harmattan, 22p.

Martinet A.C. (2008). « 50 ans de recherche en stratégie : normalisation ou pluralisme épistémologique », FCS, vol. 11, hors-série, juin.

Martinet A.C. (2009). « Management stratégique et libertés : pour une science noo-politique du concevable », Management International, vol. 13, n 3, p. 85-102. 
Martinet A.C. et Payaud M.A. (2010). « Stratégies RSE-BoP et soin des communautés humaines. Concepts et propositions génériques », Management International, vol. 14 (2), pp. 31-51.

Martinet A.C. (2012). «Albert O. Hirschman : des possibilités qui ne sont pas des nécessités... », Les Grands Inspirateurs de la Théorie des Organisations, Germain O., (coord.), Cormelles-le-Royal, EMS, p. 173-197.

Miller D. (1996). “Configurations revisited”, Strategic Management Journal, vol. 17, n $\mathrm{n}^{\mathrm{O}} 7$, p. 505-512.

Miller D. et Le Breton-Miller I. (2010). Réussir dans la Durée. Leçons sur l'Avantage Concurrentiel des Grandes Entreprises Familiales, Québec, Presses de l'Université Laval.

Morin E. (1990). Science avec Conscience, Paris, Le Seuil-Points.

Nag R., Hambrick D.C. et Chen M.J. (2007). "What is strategic management really ? Inductive derivation of a consensus definition of the field", Strategic Management Journal, vol. $28, n^{\circ} 9$, p. 935-955.

Nonaka I. et Zhu Z. (2012). Pragmatic Strategy. Eastern Wisdom, Global Success, Cambridge University Press, Cambridge, UK.

Sanchez R. et Heene A. (2010). «Grande théorie et théorie intermédiaire en stratégie : une perspective épistémologique », Revue française de gestion, vol. 36, n 204, p. 105-125.

Tannery F. (2014). «Régimes stratégiques », in Tannery F., Denis J.-P., Hafsi T., Martinet A.C., (coord.), Encyclopédie de la Stratégie, Paris, Vuibert, p. 969-992.

Tannery F., Denis J.-P., Hafsi T. et Martinet A.C. (coord.). (2014). Encyclopédie de la Stratégie, Paris, Vuibert.

Volberda H.W. (2004). "Crisis in strategy : fragmentation, integration or synthesis ?", European Management Review, vol. 1, n ${ }^{\circ}$ 1, p. 35-42. 